\title{
Experimental Measurements of Fuel Savings During Aircraft Wake Surfing
}

\author{
Curt Hanson ${ }^{1}$, Joe Pahle ${ }^{2}$, James Reynolds ${ }^{3}$, Stephanie Andrade ${ }^{4}$, and Nelson Brown ${ }^{5}$ \\ NASA Armstrong Flight Research Center, Edwards, California, 93523, USA
}

\begin{abstract}
The National Aeronautics and Space Administration (NASA) Armstrong Flight Research Center (Edwards, California) completed a series of research flights to better understand the challenges of aircraft wake surfing using civilian airplanes and commercial avionics. The research flights sought to demonstrate significant fuel savings by a pair of business jets engaged in automated wake surfing using commercial off-the-shelf avionics to the fullest extent possible, including a 1090-MHz Automatic Dependent Surveillance - Broadcast (ADS-B) data link. A NASA Gulfstream C-20A airplane (Gulfstream Aerospace, Savannah, Georgia) was flown as the trail airplane within the wake of a NASA Gulfstream III (G-III) airplane. This paper presents a summary of the fuel savings measured during those flights.
\end{abstract}

\section{Nomenclature}

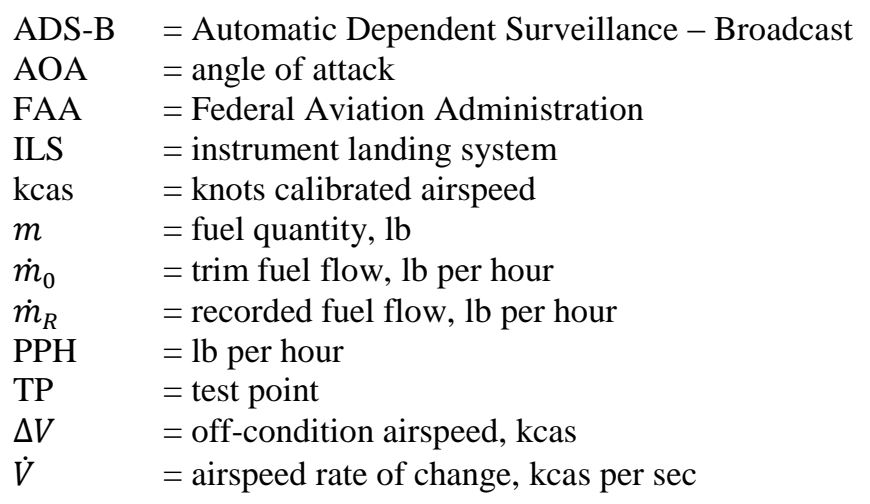

\section{Introduction}

In 2017, the National Aeronautics and Space Administration (NASA) Armstrong Flight Research Center (Edwards, California) conducted a series of flight experiments to explore the feasibility of automated aircraft wake surfing at extended trail distances while using civilian aircraft and commercial avionics, including a $1090-\mathrm{MHz}$ Automatic Dependent Surveillance - Broadcast (ADS-B) data link. The tests were performed at a trail distance of 4,000 ft, or approximately 50 wingspans. The time-in-trail at this distance was just under six seconds. Wake surfing is a method for extracting energy from the trailing wingtip vortices of another airplane (Ref. [1]). The energy is extracted by placing the wingtip of the trail airplane in the upwash portion of the vortex. The vortex upwash reduces the induced drag (drag due to lift) of the wing.

Beukenberg and Hummel (Refs. [2,3]) were the first to experimentally demonstrate fuel savings through wake surfing. Their experiments used straight-wing, propeller-driven aircraft in close formation flight - fewer than three wingspans of longitudinal separation measured from wingtip to wingtip. NASA expanded on these results with fighter aircraft, also in close formation flight (Ref. [4]), as did the United States Air Force (USAF) (Ref. [5]). NASA also investigated the wake surfing benefits of a small fighter airplane behind a large transport (Ref. [6]). In partnership

\footnotetext{
${ }^{1}$ Aerospace Engineer, Flight Controls and Dynamics Branch, P.O. Box 273, M/S 4840D, AIAA Member.

${ }^{2}$ Aerospace Engineer, Flight Controls and Dynamics Branch, P.O. Box 273, M/S 4840D, AIAA Senior Member.

${ }^{3}$ Aerospace Engineer, Flight Controls and Dynamics Branch, P.O. Box 273, M/S 4840D, AIAA Non-Member.

${ }^{4}$ Aerospace Engineer, Flight Controls and Dynamics Branch, P.O. Box 273, M/S 4840D, AIAA Member.

${ }^{5}$ Aerospace Engineer, Flight Controls and Dynamics Branch, P.O. Box 273, M/S 4840D, AIAA Non-Member.
} 
with the USAF, the Air Force Research Laboratory (AFRL), and the Defense Advanced Research Projects Agency, NASA demonstrated wake surfing benefits for large military transport aircraft in an extended formation configuration (Ref. [7]). Extended formations are those in which the aircraft are separated in the streamwise direction by more than 10 wingspans (Ref. [8]). The Boeing Company (Chicago, Illinois) and AFRL further investigated wake surfing for large military transports at extended trail distances (Ref. [9]).

For pairs of similar aircraft, these wake surfing flight experiments have shown fuel savings of $9 \%$ to $14 \%$ for close formation flight and $7 \%$ to $10 \%$ for extended formations. In all of these experiments, the trail aircraft was either under the control of the pilot by way of visual cues to the lead airplane, controlled by an autopilot with the aid of a specialized data link for transmitting position and velocity information from the lead airplane to the trail airplane, or, in some cases, controlled by a pilot using cockpit displays connected to a specialized data link.

This paper presents performance benefits for a business jet surfing the wake of a similar airplane at extended in-trail distances using two-axis automatic control and a commercial ADS-B data link for wake-relative navigation. Section III presents a description of the flight experiment, including the test aircraft, research instrumentation, test methodology, and a summary of the completed test points. Section IV introduces the methodology used to estimate fuel flow savings from experiment measurements. Section V presents the flight experiment results.

\section{Flight Experiment Description}

In 2017, NASA flew a series of four research flights with a Gulfstream C-20A airplane performing wake surfing behind a Gulfstream G-III airplane. The trail airplane was equipped with an experimental programmable autopilot that controlled lateral and vertical position relative to an estimate of the location of the wake of the lead airplane. Along-track position (distance in trail) to the lead airplane was manually controlled by pilot throttle inputs. Tablet computers mounted on the pilot's and co-pilot's yoke displayed throttle command cues, along-track command, range and error, as well as the predicted wake location. See Reynolds (Ref. [10]) for a detailed description of the pilot displays used for this flight experiment.

\section{A. Aircraft Descriptions}

The two aircraft used for the wake surfing experiment are shown in Fig. 1. The C-20A airplane is a military variant of the G-III airplane. The main differences between the lead airplane and the trail airplane are avionics modifications and external configuration. The G-III lead airplane interior and exterior were typical business jet configuration.

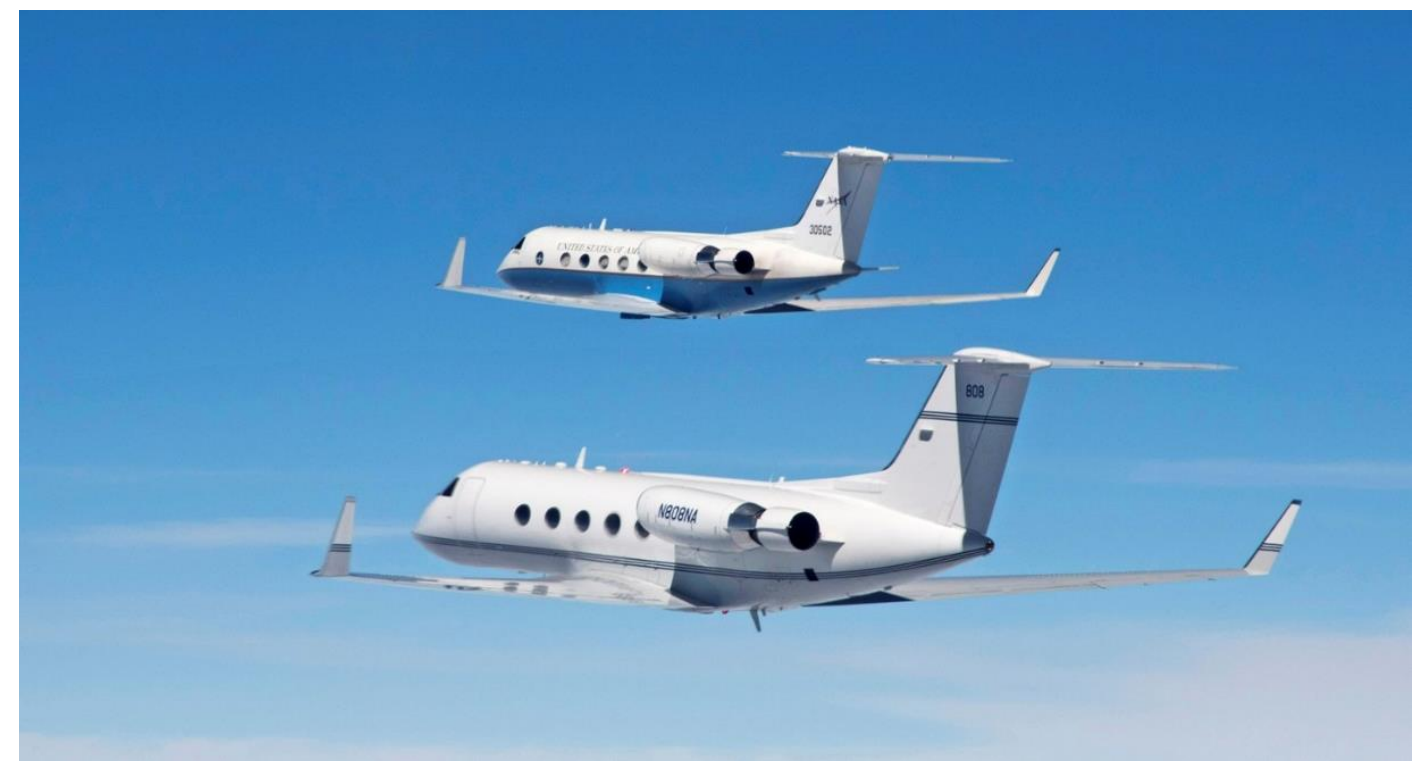

Fig. 1. NASA C-20A (white/blue) and G-III (all white) aircraft in close formation flight.

The trail airplane has been modified to carry an experimental sensor pod on the centerline below the wing. While the pod was removed for the wake surfing research flights, the pylon mount remained installed and increased fuel consumption when compared to the lead airplane at similar flight conditions and gross weights. The interior cabin of 
the C-20A airplane was in an experimental configuration, with multiple equipment racks located on both the left and right sides of the cabin.

\section{B. Experiment Systems}

The C-20A production avionics did not include an ADS-B In receiver or the necessary wake surfing navigation and control algorithms, so an experimental programmable autopilot was installed in the cabin of the trail airplane. The programmable autopilot served the function of a prototype flight director computer (FDC) modified for wake surfing, without requiring hardware and software modifications to the existing FAA-certified FDC.

The programmable autopilot used a commercially-available 1090-MHz ADS-B In receiver as a one-way data link from the lead airplane. Messages received from all nearby aircraft were filtered by International Civil Aviation Organization identifier to retain only those received from the lead aircraft. The position and velocity information in those messages, transmitted twice per second, was combined with similar information from the trail airplane to compute a relative navigation solution between the two airplanes. The ADS-B message structure uses a complex encoding scheme to achieve a resolution of 5.1 meters (at the equator) for latitude and longitude despite allocating only 17 bits to each parameter. Barometric pressure altitude is encoded with a resolution of $25 \mathrm{ft}$. The accuracy of the information contained within the ADS-B messages is dependent upon the quality of the navigational systems of the transmitting aircraft.

The horizontal location of the part of the wake nearest to the trail airplane was calculated from the reported location of the lead airplane, the time in trail, and wind estimates from the navigation system of the trail airplane. The altitude of the nearest portion of the wake is similarly found by adjusting the reported altitude of the lead airplane for the wake's assumed descent rate (Ref. [11]) and the time in trail. Figure 2 shows an example prediction of the wake location for a $10-\mathrm{kn}$ crosswind and $4,000 \mathrm{ft}$ in trail at $400 \mathrm{kn}$.

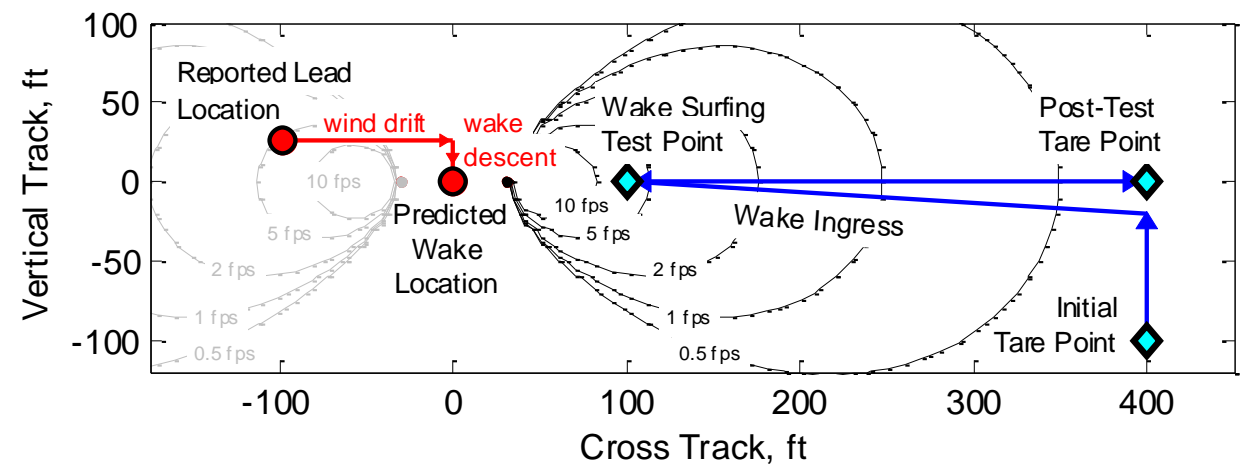

Fig. 2. Vortex upwash map with wake prediction and flight test points.

Three-axis control commands were calculated to maintain a parallel trajectory at a specified radial distance from the wake and trailing distance from the lead airplane. Cross-track and vertical-track commands were sent to the production autopilot of the trail airplane as direct current analog ILS localizer and glideslope commands, while throttle cues were given to the pilot to maintain along-track distance from the lead airplane.

\section{Test Instrumentation}

Avionics bus data were collected for the trail aircraft during the test flights, including altitude, airspeed, winds, position and velocity, control surface positions, pitch and roll rate, and Euler angles. Airspeed, winds, position and velocity, and roll angle were also recorded from the avionics bus of the lead airplane. Both aircraft were equipped with independent global positioning system (GPS) receivers for making post-flight, high-precision calculations of relative position and velocity. Control position transducers were installed and calibrated to measure the deflections of both ailerons, both spoilers, the rudder, elevator, and horizontal stabilizer on the trail airplane.

Digital fuel flow was not available for on-board recording without breaking into the production aircraft systems, so a small, portable video camera with internal recording was installed on the cockpit console with a view of the left and right engine fuel flow gauges. Just prior to takeoff, the internal clock on the camera was synchronized with a GPS time source. Recording start and stop times were also noted for post-flight correlation of the video to other on-board data sources. The camera was set to record 30 frames per second at a resolution of $1280 \times 720$. The fuel flow gauges are analog and digital readouts, with a digital resolution of $10 \mathrm{lb}$ per hour (PPH). Figure 3 shows a still shot from the 
recorded video. A character recognition software tool proved unreliable at translating the video-captured readings, so fuel flow readings for both engines at each second were transcribed from the video by hand.

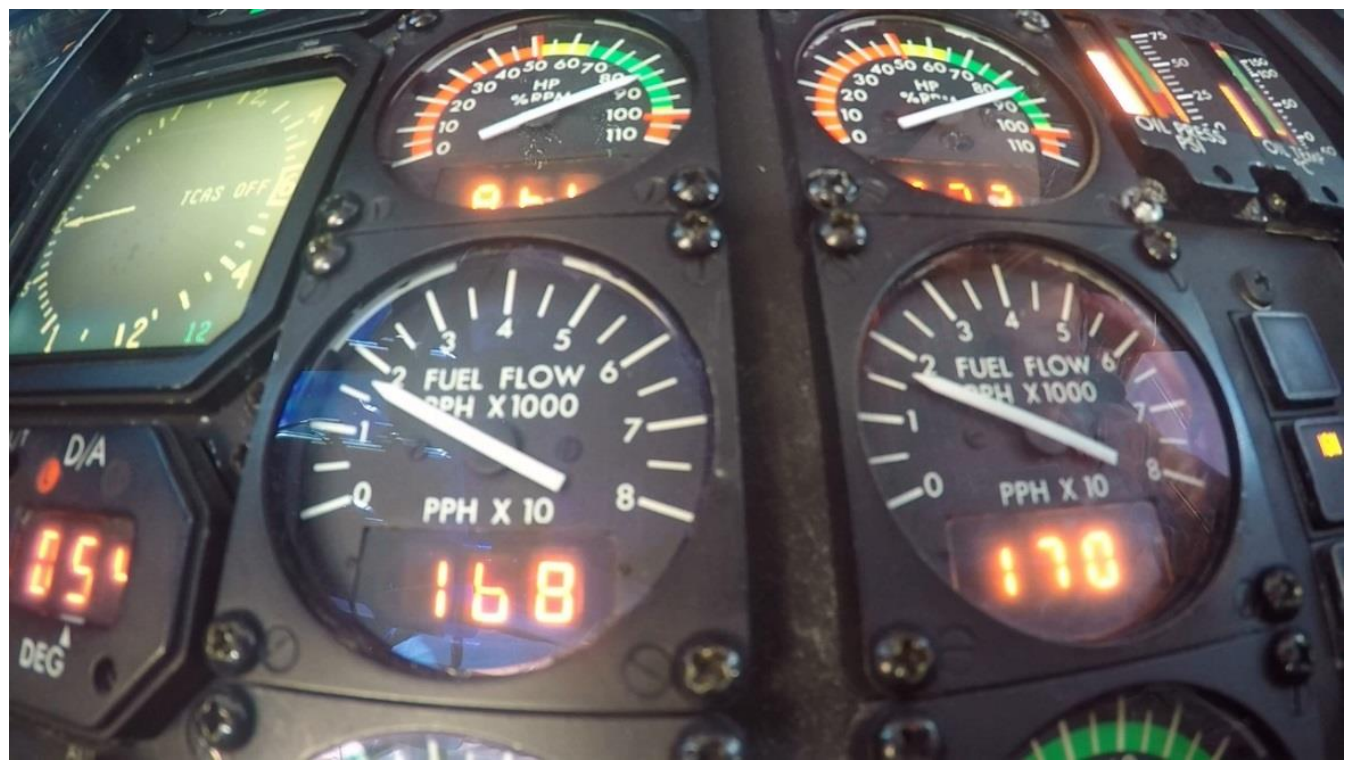

Fig. 3. Cockpit video camera view of fuel flow gauges.

At various times during each test point, pilot call-outs of fuel quantities from both airplanes were recorded by hand. The pilots' fuel quantity readings have a resolution of $100 \mathrm{lb}$.

\section{Test Methodology}

All wake surfing test points were flown at a Mach number of 0.7 and an altitude of 35,000 ft in the United States Navy W-291 test range over the Pacific Ocean off the coast of Southern California. This flight condition was chosen as approaching cruise conditions while still providing sufficient structural margin on winglet and tail loads in the event of an inadvertent wake crossing. Three-axis steering cues were provided to the trail airplane test pilot to maneuver into the desired position relative to the wake of the lead airplane prior to engaging the research system. The research autopilot was engaged approximately $4000 \mathrm{ft}$ aft of the lead airplane and outside of the predicted region of wake effects. The in-trail distance of $4000 \mathrm{ft}$ was chosen for all of the wake surfing tests because it equates to approximately 50 wingspans, which is at the larger end of the presumed usable range of extended formation flight wake surfing (Ref. [8]).

Following engagement, the pilot of the trail airplane maintained the desired along-track spacing to the lead airplane through manual throttle adjustments, based on position cues from the research system displayed on a tablet computer mounted to the control yoke. The research autopilot maintained the desired cross-track and vertical position based on own-ship data and ADS-B information transmitted from the lead airplane. The experiment test conductor entered new position commands to the research autopilot by way of a laptop computer. As shown in Fig. 2, wake surfing flight test points generally followed the process of:

1) an initial tare point,

2) systematic wake ingress,

3) stabilized wake surfing for performance and ride quality characterization, and

4) a post-test tare point.

The locations depicted in Fig. 2 represent the centerline locations of the aircraft and wake, not the wingtip or vortex core. During tare points, the team gathered a minimum of three minutes of data while stabilized outside the wake area of influence. Wake ingress was initiated when the test conductor commanded the trail airplane to a position within the wake effects and allowed the dynamics to stabilize. New position commands were subsequently chosen to incrementally move deeper into the wake. Once the experimental autopilot's steady-state roll trim surpassed a pre-specified criteria, or a significant change in ride quality was reported by the researchers in the cabin of the trail airplane, the current position command was maintained while wake surfing performance and ride quality 
measurements were collected. Wake surfing dwell times were a minimum of five minutes. Following the wake surfing points, the trail airplane was commanded out of the wake so that a post-test tare point could be completed.

\section{E. Summary of Completed Test Points}

The first three wake surfing flights were focused on developing test techniques, adjusting gains to improve stability and system tracking performance in wake effects, refining the pilot throttle cue display, and gathering measurements to characterize the wake field based on the trim state of the trail airplane at different locations within the wake. The purpose of the final flight was to gather wake surfing performance and ride quality data. In all, the project completed 17 tare points, 30 wake ingress sequences, and 10 stabilized wake surfing points.

This paper presents an evaluation of the fuel flow measurements taken during the seven wake surfing test points on the final flight and the associated tare points. See Reference [12] for an analysis of the effects of wake surfing on passenger discomfort during this experiment.

\section{Analysis Methodology}

The primary wake surfing performance measure of interest is change in trimmed fuel flow. Fuel flow varies with fuel quantity $m$ in addition to the trim state of the airplane. The trimmed fuel flow $\dot{m}_{0}(m)$ was estimated post-flight by correcting the flight-recorded fuel flow $\dot{m}_{R}(m)$ for the effects of off-condition airspeed $\Delta V$ and non-zero airspeed rate $\dot{V}$, as shown in Eq. (1).

$$
\widehat{\dot{m}}_{0}(m)=\dot{m}_{R}(m)-\left(\frac{\partial \dot{m}}{\partial V}\right) \Delta V-\left(\frac{\partial \dot{m}}{\partial \dot{V}}\right) \dot{V}
$$

The partial derivatives in Eq. (1) were estimated for the trail airplane from flight data over periods of constant throttle setting during tare test points. The pilot of the trail airplane attempted to maintain along-track spacing to the lead airplane with as few throttle adjustments as possible. Along-track error between the airplanes was allowed to vary within a range of $\pm 250 \mathrm{ft}$ because the wake effects on the trail airplane change slowly in that dimension. The large tolerance on along-track error frequently allowed for periods of constant throttle settings of 60 seconds or more.

\section{A. Off-Trim Airspeed Correction}

During all test points, the pilot of the lead airplane flew with altitude-hold and Mach-hold autopilot modes engaged. Variations in the lead airplane autopilot's performance and in changing atmospheric conditions produced small deviations from the desired airspeed condition for the test. The off-condition (off-trim) airspeed of the trail airplane at multiple analysis points during each test point is shown in Fig. 4.

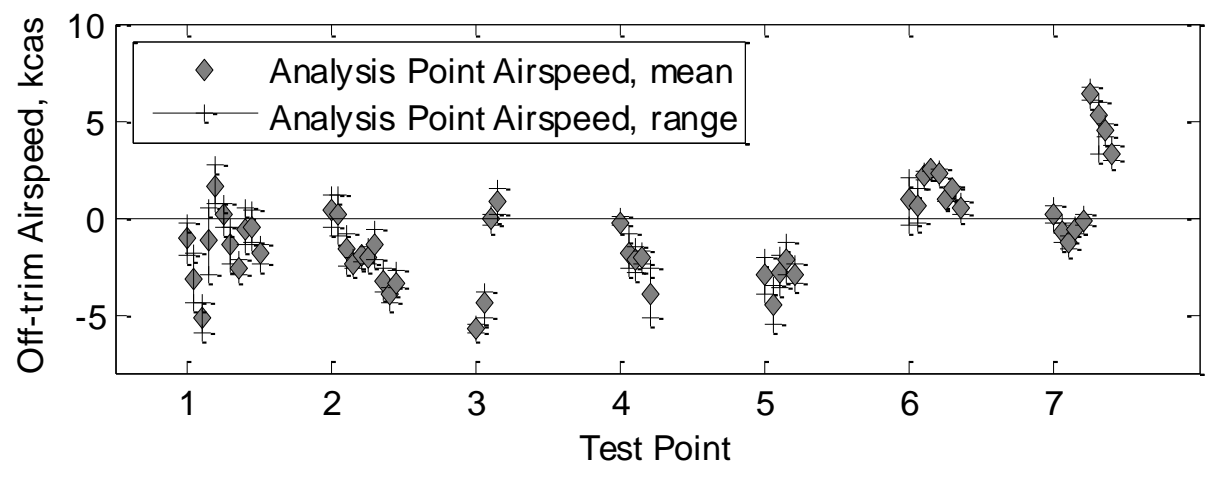

Fig. 4. Off-trim airspeed during tare test points.

A value of $16.5 \mathrm{PPH}$ per knot of airspeed variation is given for $\frac{\partial \dot{m}}{\partial V}$ in Eq. (1) by the C-20A flight manual (Ref. [13]) for a mid-fuel condition. An estimate of $\frac{\partial \dot{m}}{\partial V}$ was also derived from flight data as $13.4 \pm 4.2 \mathrm{PPH}$ per kn. Using the flight manual value, fuel flow readings taken from the video were corrected to a common reference airspeed of $235 \mathrm{kcas}$, corresponding to the intended test flight condition. The fuel flow values shown in the remainder of this paper have been corrected for airspeed. 


\section{B. Airspeed Rate Correction, Tare Points}

The test technique emphasis on minimizing throttle changes resulted in the trail airplane either gradually accelerating or decelerating during most of the test points. The zero-acceleration fuel flow correction for each tare point was estimated from multiple time segments of constant fuel flow and constant airspeed rate $\dot{V}$. An example of fuel flow measurements plotted against $\dot{V}$ for these time segments is shown in Fig. 5.

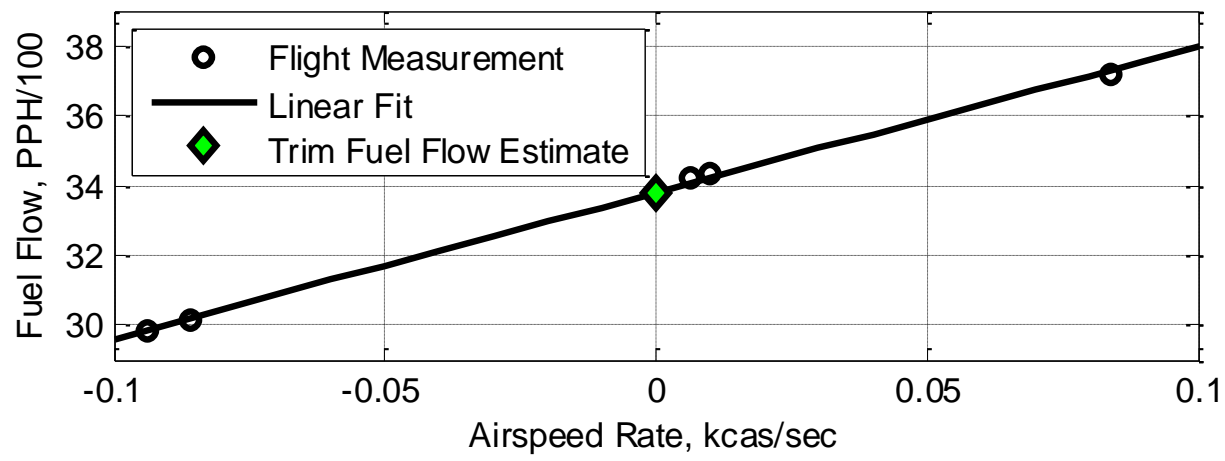

Fig. 5. Example plot for determining tare point trim fuel flow.

Assuming a linear variation of fuel flow with airspeed rate, the value of $\frac{\partial \dot{m}}{\partial \dot{V}}$ in Eq. (1) is the slope of the linear fit. Trim fuel flow estimates were calculated for each of the 11 tare test points on the final test flight. These are plotted versus total fuel quantity of the trail airplane in Fig. 6. A quadratic curve was fit to the estimates to allow for a comparison of fuel flow in and out of wake effects at any point during wake surfing tests. Uncertainty bounds for each fuel flow estimate are derived from the standard deviation of the linear fit, the standard deviation of the variations in raw fuel flow readings taken from the video, and the resolution of the fuel flow gauges.

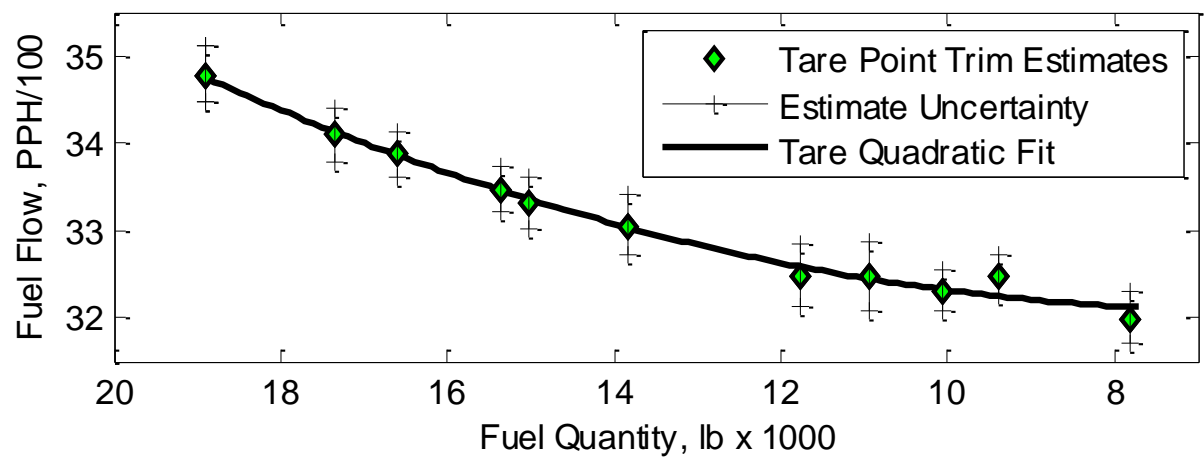

Fig. 6. Estimated fuel flow versus fuel quantity curve, tare test points.

\section{Airspeed Rate Correction, Wake Surfing}

Due to the dynamic nature of flight in vortex effects, measurements taken during wake ingress and wake surfing test points contained very few time segments of constant airspeed rate, even at constant throttle settings. Small position changes within the wake field affect the trim state of the airplane, leading to non-constant $\dot{V}$. Therefore, it would be useful to extrapolate the values of $\frac{\partial \dot{m}}{\partial \dot{V}}$ found during the tare point analysis to fuel flow measurements recorded during the wake ingress and wake surfing test points.

In order to apply the tare point $\frac{\partial \dot{m}}{\partial \dot{V}}$ values to flight in the wake, it was first necessary to determine whether fuel quantity must be taken into account. All of the tare point time segments of constant fuel flow and constant airspeed rate used to calculate values for $\frac{\partial \dot{m}}{\partial \dot{V}}$ are plotted in Fig. 7, with the off-trim fuel flow calculated by subtracting the quadratic tare estimates from the fuel flow measurements. All of the tare points show good agreement, indicating that the airspeed rate correction does not vary with fuel quantity. 


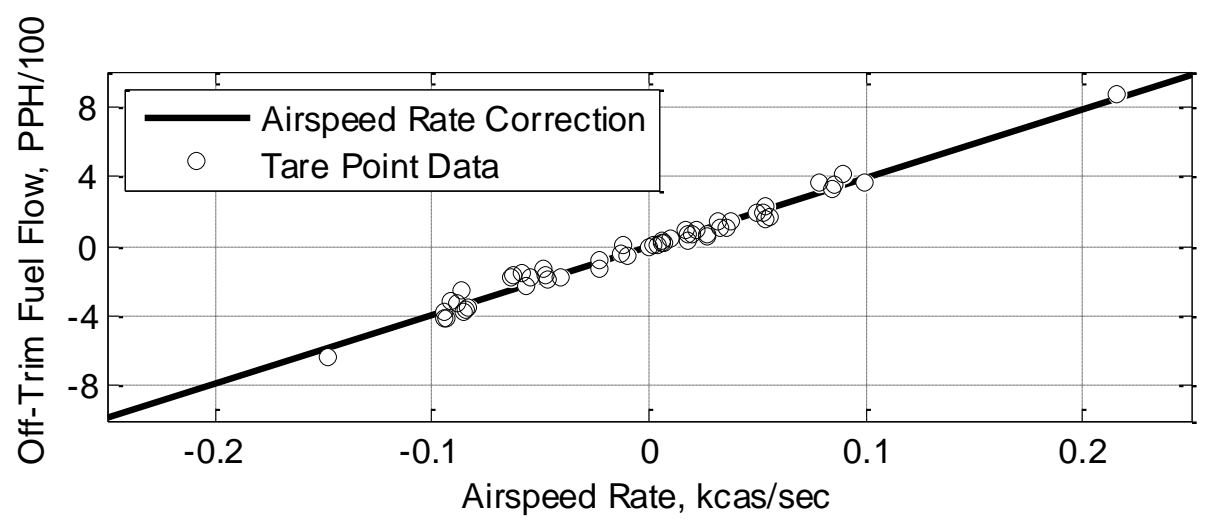

Fig. 7. Off-trim fuel flow versus airspeed rate, tare test points.

Next, a determination was made as to whether wake effects alter the value of $\frac{\partial \dot{m}}{\partial \dot{V}}$. Several time segments were identified with constant fuel flow and constant airspeed rate while in wake effects. Figure 8 shows an example of a wake surfing test point during which two fuel flow estimates were made using values of $\frac{\partial \dot{m}}{\partial \dot{V}}$ found from a linear fit of in-wake steady-state time segments. Fuel flow estimates for these segments were also calculated using the tare point value of $\frac{\partial \dot{m}}{\partial \dot{V}}$ found from the slope of the linear fit in Fig. 7. The two approaches show good agreement, indicating that wake effects do not significantly change the value of the airspeed rate correction, and that the tare point value from Fig. 7 can be applied to wake ingress and wake surfing test data.
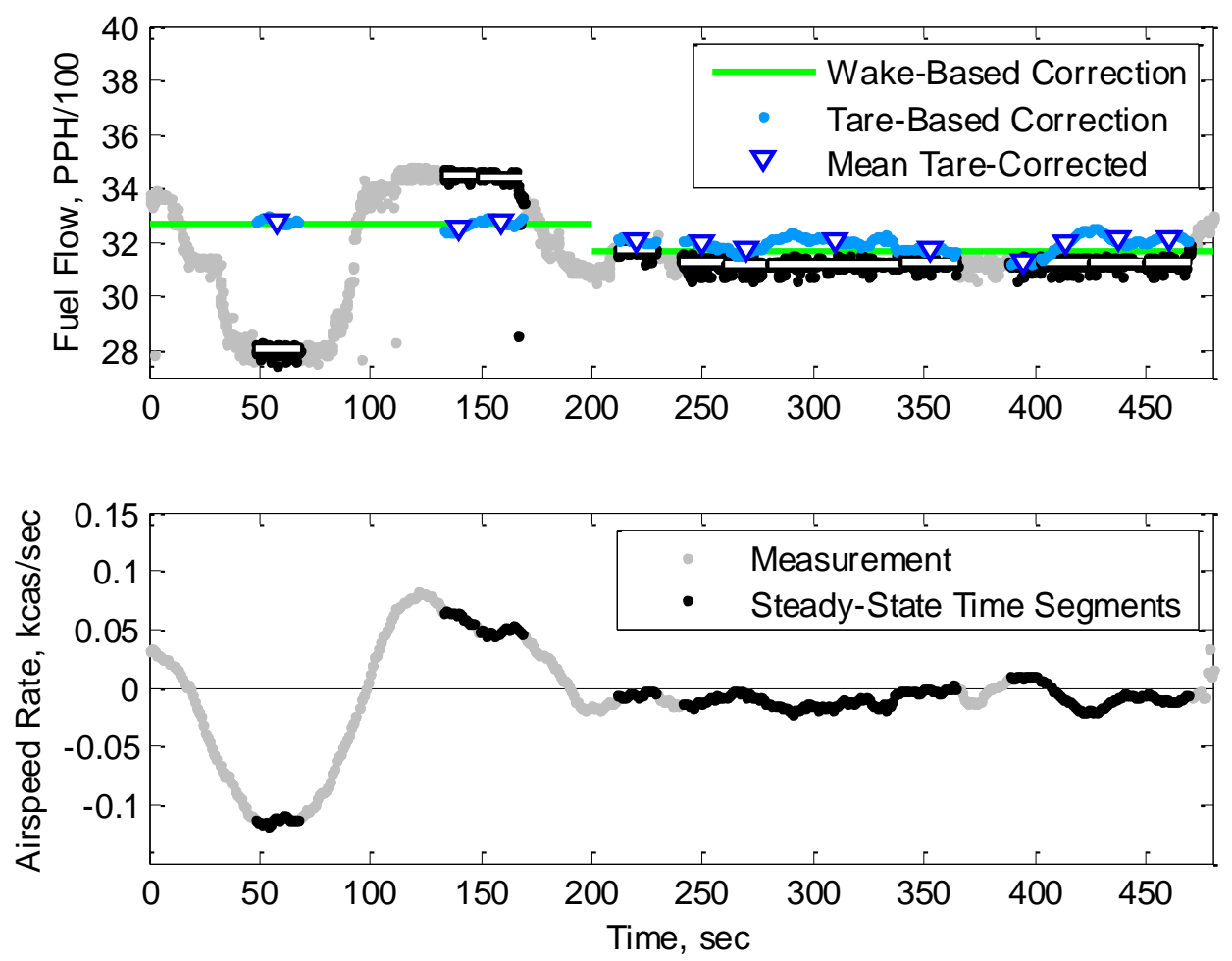

Fig. 8. Comparison of in-wake and tare-point -derived fuel flow corrections.

\section{Wake Surfing Performance}

Using the methods described above, comparisons between the corrected fuel flow measurements for the trail airplane during tare points and while immersed in wake effects provide a measure of the benefits wake surfing. The 
wake benefit predictions are then correlated with changes in trim pitch angle and roll trim changes due to the influence of the wake.

\section{A. Fuel Flow Reduction}

The percent reduction in fuel flow as compared to the tare curve was calculated for those portions of the wake ingress and wake surfing maneuvers having constant throttle settings. The mean corrected fuel flow for all of the constant-throttle time segments during the seven test points on the final research flight are shown in Fig. 9. For Test Point 2 and Test Point 7, fuel flow estimates were also calculated at $1 \mathrm{~Hz}$ for the constant-throttle time segments while in wake effects, and these estimates are also included in Fig. 9.

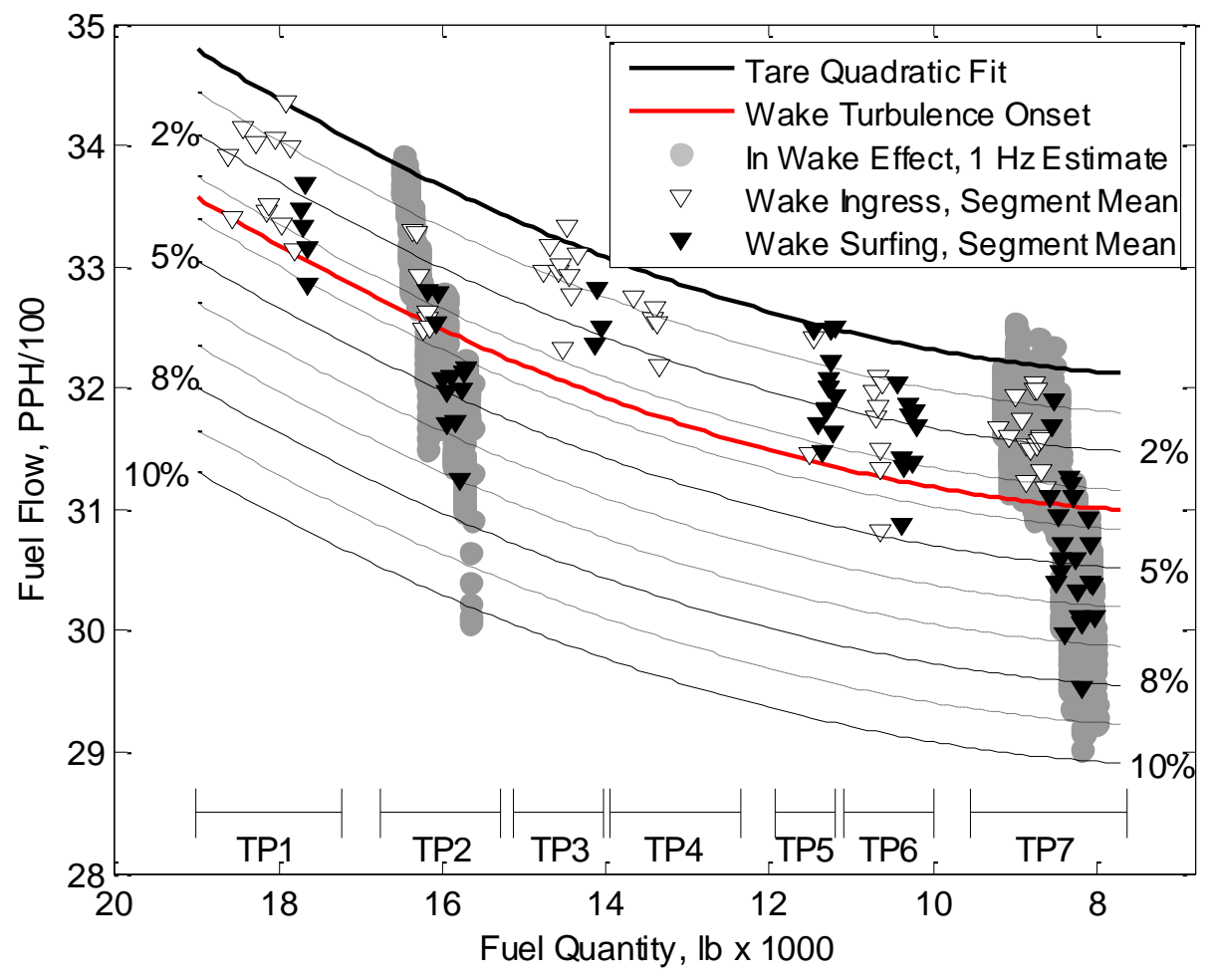

Fig. 9. Fuel flow reduction during wake ingress and wake surfing.

The wake ingress measurements were collected during the step-wise commanded approach from the tare point into the region of wake effects, and represent the steady-state fuel flow at different points within the wake. In contrast, the wake surfing measurements were taken with a constant position command to the automatic controller. However, due to the slow trim response of the controller combined with the increasingly steep trim disturbance gradient of the wake as the wingtip of the trail airplane approaches the vortex core, the wake surfing measurements were also collected at multiple locations within the wake field because the trail airplane did not consistently maintain zero tracking error. These tracking errors resulted in the wide range of fuel flow benefits measured during the wake surfing maneuvers shown in Fig. 9.

Positive fuel flow reduction in excess of two percent occurred during each of the seven wake surfing test points attempted on the final flight, although the maximum measured benefit was different in each case. The flight test procedure called for iteratively re-positioning the trail airplane deeper into the wake effects during the wake ingress maneuver until wake turbulence was felt by the cabin crew. As discussed in Ref. [12], the onset of wake-induced cabin vibration consistently occurred near 3.5-percent fuel flow reduction. As seen in Fig. 9, most of the test points stopped near this ride quality threshold.

Two of the test points failed to record a fuel flow improvement beyond the 3.5-percent ride quality threshold. Test Point 3 ended prematurely with a wake crossing event. During Test Point 4 , the cockpit fuel flow camera battery died, so the performance benefits for the wake surfing portion of that test point could not be calculated. The two test points that went significantly beyond the ride quality threshold were Test Point 2 and Test Point 7 . Both of these tests demonstrated performance benefits that varied between three-and-a-half percent and eight percent fuel flow reduction, 
with excursions as high as 10 percent. The intent of the flight experiment was to demonstrate moderate performance benefits with civilian aircraft using ADS-B for relative navigation, not to identify the maximum achievable benefit. Therefore, these results do not necessarily represent the maximum achievable benefit.

\section{B. Correlation with Wake-Relative Location}

Fuel flow reduction as a function of wake-relative cross-track and vertical-track position for Test Point 2 and Test Point 7 are shown in Fig. 10, along with theoretical predictions. Theoretical predictions of wake surfing performance benefits were calculated using a Burnham-Hallock vortex model (Ref. [14]) with a core size of one percent of the wingspan, and aerodynamic strip theory. The predictions for the two test points are slightly different due to the decreasing strength of the wake vortices as the lead airplane burned fuel and became lighter.

Test Point 2

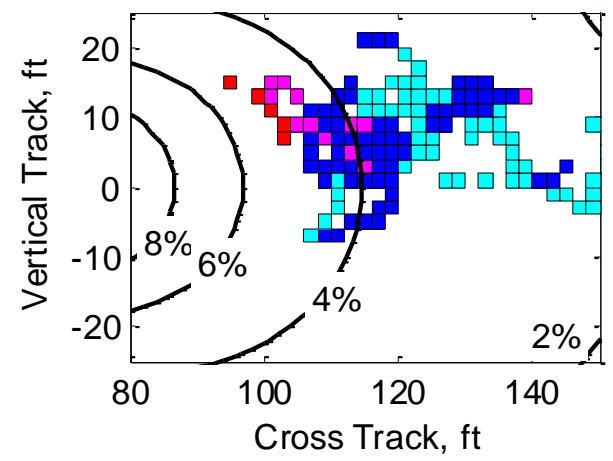

Test Point 7

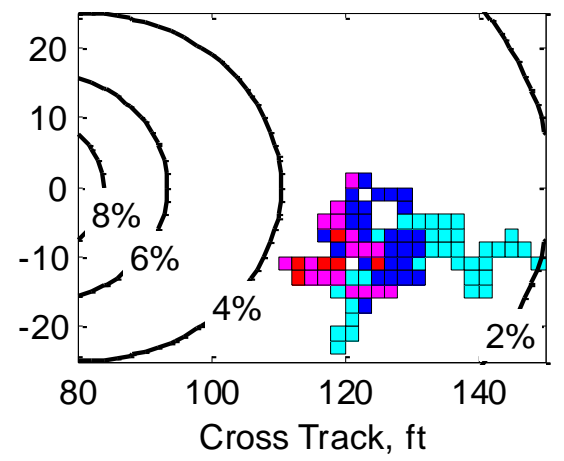

$4-6 \%$

$6-8 \%$

$8+\%$

An independent measurement of the position of the wake during the flight test was unavailable, and the accuracy of the position estimates in Fig. 10 are unknown. The wake-relative cross-track distance accuracy depends on the accuracy of the reported positions of the two aircraft, as well as the wind speed and direction reported by the trail airplane. At a time-in-trail of nearly six seconds between the two airplanes, even a one-knot error in the cross-track wind component equates to a $10-\mathrm{ft}$ position error. Wind estimate error dynamics also tend to obscure performance trends.

The accuracy of the wake-relative vertical-track distance depends on the accuracy of the reported positions of the airplanes and an assumption about the weight of the lead airplane. Real-time updates of the fuel quantity of the lead airplane were not available during the flight, so a mid-fuel weight was assumed. At the test time-in-trail, the difference in predicted wake descent distance between the heaviest (first) and lightest (last) test points was $5.2 \mathrm{ft}$. The vertical positions in Fig. 10 were corrected post-flight for lead aircraft weight.

The horizontal trends of the performance map in Fig. 10 match pre-flight predictions in that the largest benefit was measured nearest to the vortex cores. The magnitude of the measured benefit does not align well with the pre-flight predictions, likely due to errors in wind drift calculation inputs. The gradient of the flight measurements appears to be steeper than the predictions, indicating that the wake vortices may be more tightly contained than the Burnham-Hallock vortex model (Ref. [14]) predicts. Due to the narrow range of vertical positions in the test data, it is impossible to draw useful comparisons between the flight results and the pre-flight predictions for that axis.

\section{Pitch Trim Effects}

The upwash encountered by the wing of the trail airplane during wake surfing increases the local angle of attack (AOA). In order to maintain level flight and remain in formation with the lead airplane, the trail airplane must re-trim at a lower overall AOA. Measurements of AOA changes on the trail airplane during wake surfing should correlate with fuel flow reduction.

Angle of attack was not directly measurable on the trail airplane for this flight experiment. As an alternative in level flight, pitch angle can be used to approximate AOA and was recorded during the testing. Figure 11 shows the flight-measured change in pitch angle during Test Point 2 and Test Point 7 along with the change in AOA predicted 
by theory. Theoretical predictions for wake-induced changes in AOA were calculated using the same method as the wake surfing performance predictions above.

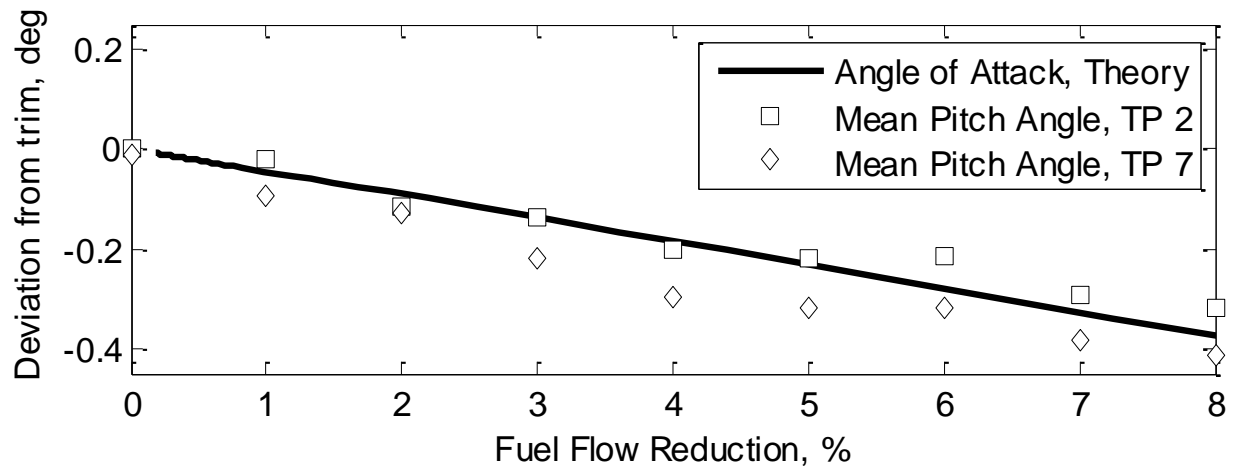

Fig. 11. Pitch angle trim effects during wake surfing.

The measured changes in trim pitch angle during wake surfing follow the trend predicted by theory. Test Point 7 consistently exhibited larger trim changes than Test Point 2, although the theoretical predictions for the different weights of the two test points were nearly identical when plotted against fuel flow reduction. In general, both test points showed reductions in trim AOA consistent with the measured reductions in fuel flow.

\section{Roll Trim Effects}

Due to the asymmetric nature of the wake vortex upwash field, the trail airplane must apply steady-state roll trim to maintain cross-track position within the wake. In the C-20A experiment, the programmable autopilot's roll commands were routed through the production ILS localizer autopilot to the ailerons and spoilers. Figure 12 shows the average aileron and spoiler deflections for varying levels of fuel flow reduction during Test Point 2 and Test Point 7.
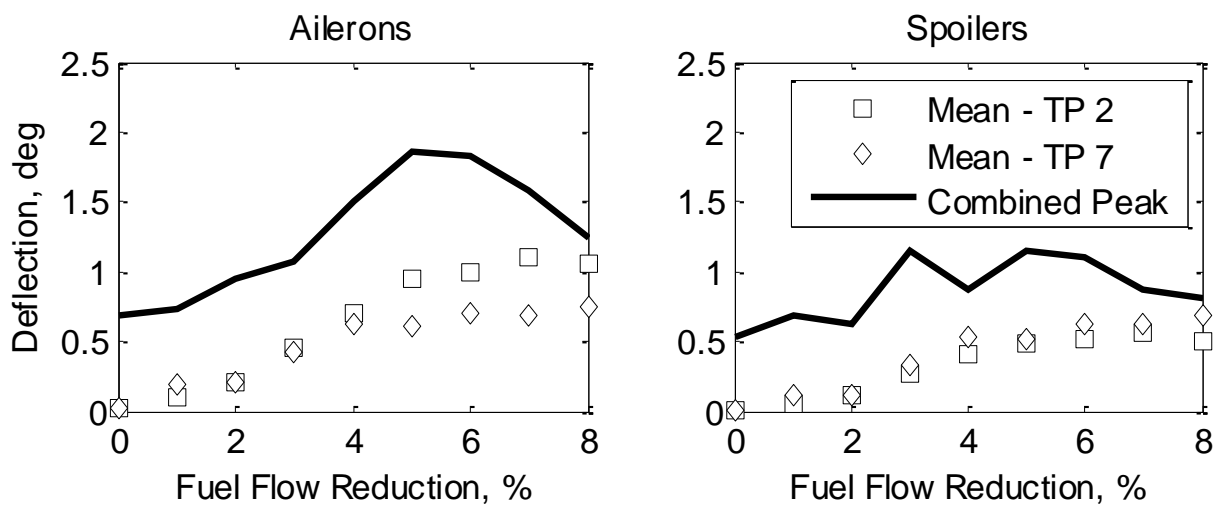

Fig. 12. Roll trim during wake surfing.

There is a clear correlation between mean roll-surface deflection and percent fuel flow reduction. This correlation is consistent with prior flight research that has shown that increases in upwash velocity at the wingtip during wake surfing are accompanied by increased roll trim requirements (Refs. [7, 9]). The maximum of the peak aileron deflection curve occurs in the region of 5 percent to 6 percent and is likely due to a combination of trim requirements and tracking error correction as the airplane was periodically disturbed from its commanded position within the wake.

Overall, the wake-induced roll trim measured during the C-20A wake surfing test points were quite small, and consistent between test points. The fuel flow penalty associated with the roll trim during these tests was not deduced from the test measurements. 


\section{Conclusion}

Performance measurements were collected for a Gulfstream C-20A airplane (Gulfstream Aerospace, Savannah, Georgia) surfing the wake of a similarly-sized Gulfstream III (G-III) airplane. The C-20A trail airplane was equipped with a programmable autopilot to predict and control the position of the airplane within the wake. Reductions in fuel flow of more than two percent were consistently measured during aircraft wake surfing when compared to fuel flow during out-of-wake tare points. Two of the test points demonstrated sustained fuel flow reduction ranging between three-and-a-half percent and eight percent, with momentary savings as high as ten percent. Wake surfing performance data were collected at the onset of wake-induced vibrations in the cabin of the trail airplane, potentially limiting the measured benefits to less than what might have been observed at stronger locations in the wake. These results help demonstrate the suitability of civilian aircraft and avionics systems, including Automatic Dependent Surveillance Broadcast (ADS-B), for operational wake surfing applications.

Additional flight research is required to mature wake surfing for commercial operations. A more comprehensive study of the effects of wake surfing on passenger ride quality is critical, as is the development of robust algorithms for wake estimation, performance optimization, and to prevent inadvertent wake crossings. New sensor technologies may also enable future wake surfing systems. There also remains work to be done on the cooperative routing and scheduling of aircraft formations, and on changes to air traffic control operations and aviation regulations.

\section{Acknowledgements}

This work was performed under the NASA Transformative Tools and Technologies project, and the NASA Flight Demonstrations and Capabilities project. The Gulfstream C-20A test airplane is maintained and operated by the NASA Science Mission Directorate.

\section{References}

[1] Wieselsberger, C., "Contribution to the Explanation of Angled Flight Patterns of Some Migratory Birds," Magazine for Flight Engineering and Motorized Aeronautics, Vol. 5, No. 15, 15 August 1914.

[2] Beukenberg, M., and Hummel, D., “Aerodynamics, Performance and Control of Airplanes in Formation Flight," ICAS-90-5.9.3, 1990.

[3] Hummel, D., "The Use of Aircraft Wakes to Achieve Power Reductions in Formation Flight," in AGARD CP-584, 1996, pp. 36-1 - 36-13.

[4] Vachon, M. J., Ray, R. J., Walsh, K. R., and Ennix, K., "F/A-18 Aircraft Performance Benefits Measured During the Autonomous Formation Flight Project," AIAA 2002-4491, 2002. doi: $10.2514 / 6.2002-4491$

[5] Wagner, Maj. G., Jacques, Lt. Col. D., Blake, W., and Pachter, M., "Flight Test Results of Close Formation Flight for Fuel Savings," AIAA 2002-4490, 2002. doi: $10.2514 / 6.2002-4490$

[6] Johnsen, F. A., "NASA Sky Surfing for Fuel Economy,” NASA News Release 03-42, July 21, 2003.

[7] Pahle, J., Berger, D., Venti, M., Duggan, C., Faber, J., and Cardinal, K., “An Initial Flight Investigation of Formation Flight for Drag Reduction on the C-17 Aircraft," AIAA 2012-4802, 2012. doi: 10.2514/6.2012-4802

[8] Ning, A. S., Flanzer, T. C., and Kroo, I. M., “Aerodynamic Performance of Extended Formation Flight,” Journal of Aircraft, Vol. 48, No. 3, May-June 2011, pp. 855-865. doi: 10.2514/1.C031046

[9] Bieniawski, S. R., Clark, R. W., Rosenzweig, S. E., and Blake, W. B., "Summary of Flight Testing and Results for the Formation Flight for Aerodynamic Benefit Program," AIAA 2014-1457, 2014. doi: 10.2514/6.2014-1457

[10] Reynolds, J., Pahle, J., Asher, T., and Hanson, C., "Design, Development, and Flight Evaluation of Pilot Displays and Long Track Control for Wake Surfing Applications," to be presented at the AIAA 2018 Flight Testing Conference, Atlanta, Georgia, June 25-29, 2018 (to be published).

[11] Brashears, M. R., and Hallock, J. N., “Aircraft Wake Vortex Transport Model,” AIAA-73-679, 1973. doi: 10.2514/6.1973-679

[12 Hanson, C., Andrade, S., and Pahle, J., "Experimental Measurements of Passenger Ride Quality During Aircraft Wake Surfing," to be presented at the AIAA 2018 Flight Testing Conference, Atlanta, Georgia, June 25-29, 2018 (to be published).

[13] Gulfstream Aerospace Corp., "Flight Manual, Performance Data, USAF C-20A/C-20B Aircraft, TO 1C-20B-1-1 Change 8," September 15, 2004.

[14] Burnham, D. C., and Hallock, J. N., "Chicago Monostatic Acoustic Vortex Sensing System, Volume IV: Wake Vortex Decay," DOT/FAA/RD-79-103, IV, 1982. 